\title{
AVALIAÇÃO EM MASSA DE IMÓVEIS USANDO REGRESSÃO ESPACIAL E KRIGAGEM BAYESIANA (UM ESTUDO DE CASO EM BALNEÁRIO CAMBORIÚ/SC)
}

\author{
Mass evaluation in real estate using geostatistics and Bayesian Kriging (a case \\ study in Balneário Camboriú/SC)
}

\author{
Ricardo André Hornburg', Norberto Hochheim²
}

Recebido em 14 de julho de 2016; recebido para revisão em 31 de outubro de 2016; aceito em 17 de dezembro de 2016; disponivel on-line em 07 de março de 2017.

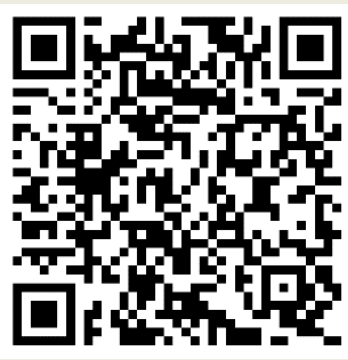

PALAVRAS CHAVE:

Planta de valores genéricos;

Avaliação em massa;

Estatística bayesiana;

Krigagem.

\section{KEYWORDS:}

Standard ground value; Bulk value appraisal; Bayesian statistics; Kriging.

RESUMO: Uma das grandes dificuldades que se tem na avaliação em massa de imóveis é encontrar um modelo que mostre a realidade do mercado de imóveis para que se possa construir uma Planta de Valores Genéricos (PVG), usada como base para a cobrança do Imposto Predial e Territorial Urbano (IPTU). Este artigo apresenta um método que combina o uso da econometria espacial com a geoestatística bayesiana visando estimar o valor dos imóveis levando em consideração as interações espaciais devidas às características da localização. Os métodos da regressão espacial e da krigagem bayesiana são usados com esta finalidade. A técnica da regressão espacial possibilita a modelagem da dependência espacial. A técnica da krigagem bayesiana permite estimar valores de variáveis espacialmente distribuídas a partir de valores adjacentes considerados como interdependentes. Dessa maneira, a krigagem é considerada um método de médias móveis. O semivariograma é a ferramenta básica de suporte às técnicas de krigagem, permitindo representar quantitativamente a variação de um fenômeno regionalizado no espaço. Uma aplicação do método proposto é realizada no bairro Centro da cidade de Balneário Camboriú (SC), usandose uma amostra de dados de mercado para a avaliação em massa de imóveis do tipo apartamento. Destaca-se como contribuição do método apresentado uma possibilidade de melhora na determinação do valor justo dos imóveis numa avaliação em massa, amparando assim a equidade e consequente justiça fiscal quando aplicados pelos municípios.

ABSTRACT: One of the greatest difficulties in bulk value appraisal of buildings is to find a model that shows the reality of the real estate market so you can build a Standard Ground Value (SGV), used as a basis for the collection of Land and Territorial Tax Urban (Land Tax). This paper presents a method that combines the use of spatial econometrics with Bayesian geostatistics in order to estimate the value of real estate taking into account the spatial interactions due to the characteristics of the location. The methods of spatial regression and Bayesian kriging are used for this purpose. The spatial regression technique allows the modeling of spatial dependence. The Bayesian kriging technique allows the estimation of values of spatially distributed variables from adjacent values considered as interdependent. In this way, kriging is considered a moving average method. The semivariogram is the basic tool to support kriging techniques, allowing quantitative representation of the variation of a regionalized phenomenon in space. An application of the proposed method is carried out in the downtown neighborhood of Balneário Camboriú (SC), using a sample of market data for the mass evaluation of apartment-type properties. As a contribution of the method presented, there is a possibility of improvement in the determination of the fair value of real estate in $a$ mass valuation, thus supporting equity and consequent fiscal justice when applied by municipalities.

\footnotetext{
* Contato com os autores:

${ }^{1}$ e-mail: ricardo_andreh@yahoo.com.br (R. A. Hornburg )

Economista, Professor Doutor no curso de Engenharia Civil da Faculdade AVANTIS.

2e-mail: hochheim@gmail.com (N. Hochhaim)

Engenheiro Civil, Professor Doutor no curso de Engenharia Civil da Universidade Federal de Santa Catarina - UFSC.
} 


\section{INTRODUÇÃO}

A aprovação da lei de responsabilidade fiscal dos Estados e municípios brasileiros impôs a essas entidades políticas o controle dos gastos, condicionado à capacidade de arrecadação de tributos. Isso gerou a necessidade de um maior planejamento, em especial, no que se refere aos municípios, do imposto sobre a propriedade urbana, também conhecido como Imposto Predial e Territorial Urbano (IPTU), o qual é calculado como um percentual do valor venal dos imóveis.

$\mathrm{Na}$ avaliação dos imóveis procura-se encontrar modelos estatísticos adequados que considerem as reais condições e fatores locais do mercado imobiliário.

As variáveis encontradas para compor o valor de um determinado subconjunto de imóveis, não são necessariamente as mesmas para um outro subconjunto de imóveis, para tanto, em alguns casos deve-se retirar variáveis e acrescentar outras, pois cada subconjunto de imóveis pode ter suas próprias características que não são necessariamente iguais aos outros subconjuntos.

$\mathrm{Na}$ engenharia de avaliações, os modelos estatísticos são de suma importância para a elaboração da Planta de Valores Genéricos (PVG), que permite com que as prefeituras possam determinar o IPTU com máxima equidade.

Uma grande dificuldade que se tem na utilização de métodos econométricos, na busca de modelos de avaliação, está em considerar a variável localização que pode valorizar ou desvalorizar os imóveis.

A exclusão da localização da modelagem pode causar sérios problemas de predição, pois dados localizados espacialmente (que é o caso dos imóveis), em geral, apresentam autocorrelação ou covariância espacial. Preditores que não levam isso em consideração estão muito longe da realidade (CRESSIE, 1993).

As variáveis de localização que não são representadas e modeladas corretamente podem gerar problemas nos modelos de regressão. Além da perda do poder de explicação do modelo, pode provocar a autocorrelação espacial nos resíduos do modelo pelo tratamento incompleto ou inadequado dos fatores de localização.

Buscando soluções teóricas e de cunho metodológico para estes problemas, existem duas abordagens estatísticas diferentes para o tratamento e modelagem dos efeitos espaciais nos dados de mercado que são a econometria espacial e a geoestatística. Este trabalho mostra como estas duas abordagens podem ser usadas de forma combinada, contribuindo para uma determinação mais acurada do valor dos imóveis.

Para tanto, o objetivo colimado neste trabalho é propor um método capaz de determinar valores de mercado para avaliação em massa de imóveis, através do uso combinado da técnica da regressão espacial com a krigagem bayesiana. Depois de modelar a dependência espacial usando modelos de regressão espacial, vai-se usar os resultados para elaborar uma planta de valores genéricos para o local de estudo usando-se krigagem bayesiana.

\section{AVALIAÇÃO EM MASSA DE IMÓVEIS}

\subsection{AVALIAÇÃO IMOBILIÁRIA}

A função principal de uma avaliação é assegurar o valor de algum tipo de imóvel sob um determinado conjunto de condições. Os valores das propriedades variam consideravelmente de um local para outro (GONZÁLEZ, 2002).

Atribui-se valor a tudo que é útil ou escasso. Cabe à avaliação traduzir essa utilidade ou escassez e associar a necessidade e/ou desejo de possuir um bem numa quantia monetária (AYRES, 1996).

O valor de um imóvel depende diretamente das características do entorno, tais como: tipos de imóveis existentes, ruas, utilidades, conveniências. Além o entorno imediato, o imóvel relaciona-se com a cidade inteira. Todavia, nem o declínio econômico de uma cidade afeta todas as suas partes igualmente (CAN, 1998).

A avaliação de imóveis urbanos deve se basear na NBR 14653-2 (ABNT, 2011). Para esta norma, a avaliação de imóveis deve preferencialmente se fundamentar na pesquisa de mercado. Devem ser colhidas amostras com preços de imóveis ofertados e comercializados, além de 
atributos que afetem o valor. Tais atributos serão ponderados ou por inferência estatística ou por homogeneização. A amostra deve ser representativa do mercado imobiliário em análise.

Estudos sobre Avaliação Imobiliária, como de Cesare (2004), apontam três principais metodologias para avaliação em massa para fins fiscais: Método de Custo de Reprodução, Método de Renda e Método Comparativo de Dados de Mercado.

Para Silva (2006), o objetivo da avaliação em massa de imóveis é obter o valor de todos os imóveis localizados em determinada área. Emprega métodos que devem ter respaldo legal. A metodologia empregada deve evitar ao máximo o subjetivismo, tanto dos dados quanto dos procedimentos, além de adequar os mesmos ao mercado real (POLI et al., 2011).

Möller (1995) ressalta que a avaliação em massa de imóveis deve ser vinculada ao estudo do Código Tributário Municipal - CTM, no que se refere aos impostos sobre a propriedade (IPTU e ITBI, impostos da esfera municipal). Dessa forma, o resultado final da avaliação se adequará às exigências legais.

A avaliação em massa de imóveis geralmente é obtida pelos seguintes métodos de avaliação: método evolutivo e de comparação de dados de mercado. $O$ primeiro requer as estimativas do valor do terreno e o corrente custo de reprodução das edificações. O segundo método faz a estimativa do valor de mercado com base em preços de grupos de imóveis vendidos em um período que antecede a data de lançamento dos tributos (SILVA, 2006).

Zancan (1996) alerta que a desvantagem da metodologia usualmente utilizada na avaliação em massa, que calcula separadamente os valores do terreno e das edificações, está em não existir um mercado de edificações separado dos terrenos sobre os quais foram construídas.

\subsection{MODELOS DE REGRESSÃO ESPACIAL}

Geralmente, em uma análise de regressão procura-se encontrar um bom ajuste do modelo aos dados, no sentido de reduzir a diferença entre os valores preditos pelo modelo e os valores observados da variável dependente. Também se procura descobrir quais das variáveis explicativas contribuem de forma significativa para o relacionamento linear. Uma hipótese é que as observações não sejam correlacionadas e, portanto, os termos aleatórios (resíduos) $\varepsilon_{1}$ do modelo são independentes e não-correlacionados entre si, além de apresentar distribuição normal com média zero e variância constante, isto no modelo clássico. No caso de dados onde está presente a dependência espacial, é muito pouco provável que esta hipótese de observações não correlacionadas seja verdadeira. E no caso mais comum, os termos aleatórios continuam apresentando a autocorrelação espacial presente nos dados, que pode se manifestar por diferenças regionais sistemáticas, ou ainda por uma tendência espacial contínua (LOPES; BRONDINO; SILVA, 2006).

Segundo Serrano e Valcarce (2000), quando se trabalha particularmente com dados de natureza espacial podem aparecer os denominados efeitos espaciais como a heterogeneidade e a autocorrelação espacial. A heterogeneidade aparece quando os dados utilizados para explicar um mesmo fenômeno são de unidades espaciais muito distintas, sendo que os problemas mais frequentes são a instabilidade estrutural e a heterocedasticidade. A heterocedasticidade espacial ocorre pela omissão de variáveis ou outras formas de especificação que levam à aparição dos denominados erros de medidas. A dependência ou autocorrelação espacial surge sempre que o valor de uma variável em um lugar do espaço está relacionado com seu valor em outro ou outros lugares do espaço.

Para Paiva e Khan (2010), a Análise Exploratória de Dados Espaciais (AEDE), ou Exploratory Spatial Data Analysis (ESDA):

"é feita com base em um conjunto de ferramentas gráficas e descritivas cujo objetivo é identificar propriedades espaciais dos dados. Está baseada nos aspectos espaciais das informações, ou seja, trata diretamente de questões como dependência espacial e heterogeneidade espacial. $O$ objetivo é descrever a distribuição espacial, os padrões de 
associação espacial, verificar a existência de diferentes regimes espaciais ou outras formas de instabilidade espacial $e$ identificar agrupamento de valores semelhantes (clusters), ou de observações atípicas (outliers). O cluster espacial é um agregado de ocorrências no espaço ou a ocorrência de valores semelhantes em áreas próximas; já os outliers espaciais são dados cuja localização pode exercer uma forte influência, especialmente nas estimações."

A presença de autocorrelação espacial é medida usualmente por meio de estatísticas globais. Os indicadores globais constituem uma aproximação mais tradicional do efeito da dependência espacial, em que a estrutura geral de dependência fica resumida em um único valor. (LEMOS et al., 2005a).

Segundo Anselin (2005), a dependência espacial pode ser incorporada nos modelos clássicos de regressão de duas formas: como um regressor adicional na forma de uma variável dependente espacialmente defasada $(W y)$, ou uma estrutura espacialmente defasada no erro da regressão $(W e)$. O primeiro modelo é conhecido como modelo de defasagem espacial ou da variável dependente defasada e o segundo é o modelo do erro espacial ou do erro espacialmente correlacionado.

\subsection{MODELO DE DEFASAGEM ESPACIAL}

No modelo de defasagem espacial, em inglês SAR (Spatial Auto Regressive ou Spatial Lag Model), a autocorrelação espacial ignorada é atribuída à variável dependente $Y$, conforme expressa a Equação 1.

$$
Y=\rho W Y+X \beta+\varepsilon
$$

Em que:

$Y=$ variável dependente;

$X=$ variáveis independentes;

$\beta=$ coeficientes de regressão;

$\varepsilon=$ erros aleatórios com média zero e variância $\sigma^{2}$ constante;

$W=$ matriz de vizinhança espacial ou matriz de ponderação espacial;

$\rho=$ coeficiente espacial autoregressivo.

\subsection{MODELO DO ERRO ESPACIAL}

O modelo autoregressivo do erro, em inglês CAR (Conditional Auto Regressive ou Spatial Error Model), pode ser expressado formalmente conforme a Equação 2:

$Y=X \beta+\varepsilon, \quad \varepsilon=\lambda W \varepsilon+\xi$

Em que:

$W \varepsilon=$ erros com efeito espacial;

$\xi=$ erros aleatórios com média zero e variância $\sigma^{2}$;

$\lambda=$ coeficiente autoregressivo.

Segundo Anselin (1999a), o método de estimação dos parâmetros do modelo normalmente usado é o de máxima verossimilhança, entretanto outros métodos também têm sido propostos, por exemplo, como os de variáveis instrumentais, mínimos quadrados espaciais, método dos momentos, método dos códigos, métodos bayesianos, entre outros.

\subsection{ESTATÍSTICA BAYESIANA}

Thomas Bayes foi um reverendo presbiteriano que viveu no início do século 18 (1701-1761) na Inglaterra. Estudou teologia na Universidade de Edimburgo (Escócia). Em 1737 publicou seu primeiro e único livro de matemática, chamado The doctrine of fluxions ( $A$ doutrina dos fluxions) (PENA, 2006).

Para Gangsei (2013), a estatística bayesiana oferece alguns benefícios em comparação com métodos alternativos, sendo um deles, a capacidade de utilizar "todos" os dados disponíveis, e também a sua utilidade para lidar com dados faltantes, formando uma base adequada a fim de aproveitar uma versão ligeiramente modificada do modelo em áreas carentes em dados.

\subsubsection{Teorema de Bayes}

Este teorema é uma das pedras angulares da estatística das probabilidades combinadas, e é largamente utilizada em áreas a primeira vista pouco relacionadas, como Medicina e Informática (SORENSEN et al., 1994).

$\mathrm{Na}$ primeira área, por exemplo, o paradigma embasado em evidências é todo 
construído em cima do teorema de Bayes. Baseado na experiência acumulada de exames e testes para tentar diagnosticar uma doença, o médico enquadra seus pacientes e pode estimar qual a probabilidade de que uma dada doença esteja se manifestando. Assim, dada uma probabilidade inicial (por exemplo, o paciente é fumante) é aplicado um exame em que, se sabe, há uma probabilidade de falsos-positivos e falsos-negativos (por exemplo, uma biópsia de pulmão), o médico sabe qual a probabilidade resultante daquele paciente ter a doença (por exemplo, câncer de pulmão) (ANDRADE, 1999).

$\mathrm{Na}$ informática, muitos dos sistemas de classificação automática são baseados no teorema de Bayes. Inicialmente o sistema é treinado, aceitando entradas de humanos que dizem que uma dada entrada pertence a determinado grupo. Com o tempo, o sistema acumula um grande banco dessas informações e, aplicando o teorema de Bayes, consegue estimar a probabilidade de cada novo dado de pertencer a cada grupo já classificado (DIGGLE, RIBEIRO, 2007).

Sendo assim, pode-se dizer que o bayesianismo tem dois importantes alicerces epistemológicos: $1^{\circ}$ ) a visão do universo com base em graus de confiabilidade; 2ㅇ) uma regra matemática que explicita como você deve mudar suas crenças à luz de novos dados empíricos. A partir desses pilares se podem deduzir uma série de implicações filosóficas (PENA, 2006).

Segundo Carroll (2010), a regra de Bayes é um teorema fundamental que pode ser trivialmente derivada dos axiomas da probabilidade. Esta lei pode ser vista como a lei fundamental e universal de aprendizagem.

Wettig (2013) afirma que a regra de Bayes é a base central do raciocínio bayesiano. Trata-se de uma consequência direta da regra da cadeia de probabilidades que afirma que a probabilidade conjunta de um conjunto de variáveis aleatórias pode ser escrito como uma cadeia de probabilidades condicionais.

Para Amaral e Inácio (2010), os dois conceitos bayesianos fundamentais são: a) coisas que são desconhecidas são representadas por distribuições de probabilidade, e b) coisas que são conhecidas (dados) são usadas para aperfeiçoar o conhecimento acerca do problema, a partir do Teorema de Bayes.

Desta forma, para que se possa chegar ao teorema de Bayes, segundo Pena, (2006), parte-se dos seguintes princípios básicos:

"Assim, a probabilidade de que observemos simultaneamente um evento A e um evento $B$ é dada pela Equação 3:

$$
\mathrm{P}(\mathrm{A} \cap \mathrm{B})=\mathrm{P}(\mathrm{A} / \mathrm{B}) \cdot \mathrm{P}(\mathrm{B}) \quad \text { Eq. [3] }
$$

Por outro lado, a probabilidade de que observemos simultaneamente um evento $A$ e um evento $B$ também pode ser dada pela Equação 4:

$P(B \cap A)=P(A \cap B)=P(B / A) . P(A) \quad$ Eq. [4] Combinando (1) e (2), temos a Equação 5:

$$
\mathrm{P}(\mathrm{A} / \mathrm{B}) . \mathrm{P}(\mathrm{B})=\mathrm{P}(\mathrm{B} / \mathrm{A}) \cdot \mathrm{P}(\mathrm{A}) \quad \text { Eq. [5] }
$$

Rearranjando, chegamos ao teorema de Bayes pela Equação 6:

$$
P(A / B)=\frac{P(B / A) \cdot P(A)}{P(B)} \quad \text { Eq. [6] }
$$

Como geralmente não conhecemos $P(B)$, precisamos usar uma formulação alternativa, que é baseada em (Equação 7):

$$
\mathrm{P}(\mathrm{B})=\mathrm{P}(\mathrm{B} \cap \mathrm{A})+\mathrm{P}(\mathrm{B} \cap \mathrm{AC}) \quad \text { Eq. [7] }
$$

Em que, Ac é o evento complementar de $A$, também chamado de não-A. Usando nosso conhecimento básico (Equação 1) $e$ substituindo, obtemos a Equação 8:

$P(B)=[P(B / A) \cdot P(A)]+[P(B / A C) \cdot P(A C)]$ Eq. $[8] . "$

Substituindo a Equação 8 na Equação 6 obtem-se a formulação alternativa:

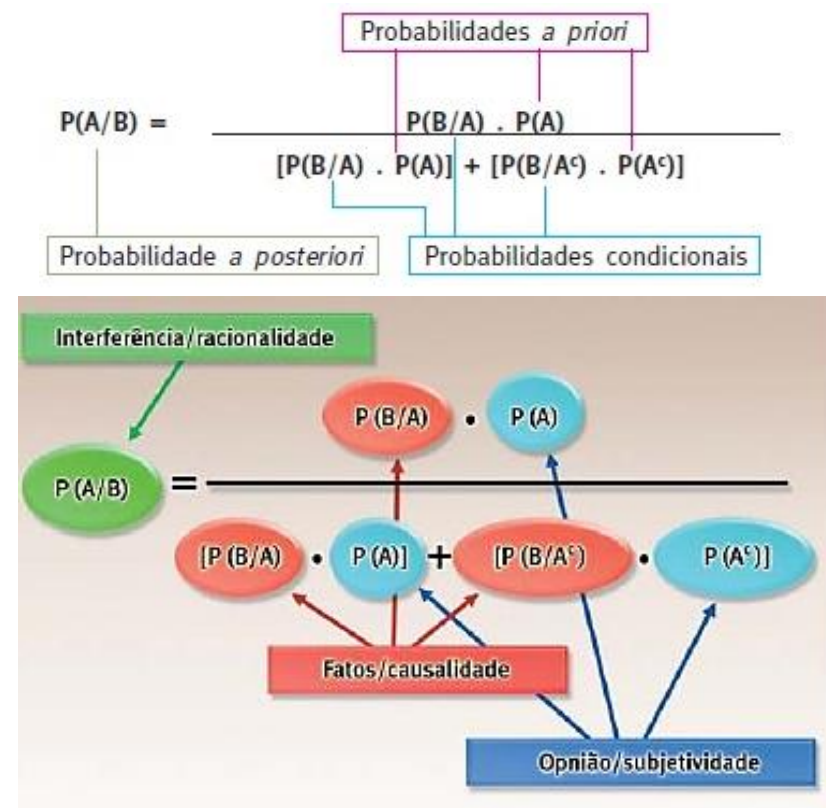

FIGURA 1: Fórmula alternativa. 
A regra de Bayes apresenta uma maneira de como alterar as probabilidades a priori considerando novas experiências para obter probabilidades a posteriori.

Para DiMaggio (2014), uma vantagem do método bayesiano é o ajuste que se pode dar nos dados espaciais usando uma distribuição a priori que irá explicar como que a correlação espacial irá se comportar no modelo para dar resultados mais precisos.

Talvez a vantagem prática mais importante da abordagem bayesiana para a aprendizagem é "o princípio da otimização bayesiana", que afirma que, quando usado em conjunto com a teoria da decisão, decisões baseadas em inferência usando a regra de Bayes sempre produzirão uma utilidade esperada igual ou maior do que as decisões com base em qualquer outra técnica (CARROLL, 2010).

A probabilidade a priori $P(A)$, segundo Amaral e Inácio (2010), fornece a probabilidade dos diferentes valores possíveis da quantidade de interesse antes dos dados serem considerados. Representa o estado do conhecimento anterior aos dados.

A priori informativa é quando se conhece alguma coisa acerca do parâmetro desconhecido $A$ ou sobre o experimento sendo realizado, usam-se essas informações no estabelecimento da função densidade de probabilidade a priori para A. Se essa densidade contiver parâmetros, estes são estabelecidos fora do modelo (hiperparâmetros) (BRASIL, 2012).

Por outro lado, a priori não informativa ocorrem em situações que se conhece muito pouco ou quando não se tem nenhuma informação disponível a priori, sendo que todos os possíveis valores de A como igualmente prováveis, isto é, com uma distribuição a priori uniforme (EHLERS, 2003).

Para Amaral e Inácio (2010), a verossimilhança $P(B / A)$, fornece a probabilidade de obter o dado, considerando diferentes valores possíveis da quantidade desconhecida de interesse (hipótese $H$ ).

A probabilidade a posteriori $\mathrm{P}(\mathrm{A} / \mathrm{B})$, fornece a probabilidade dos diferentes valores possíveis da quantidade de interesse depois de considerar os dados, representando o estado do conhecimento posterior aos dados (AMARAL e INÁCIO, 2010).

Em resumo, a probabilidade a posteriori é a combinação da priori com a verossimilhança, sendo que a diferença entre priori e a posteriori é o aprendizado que se obtêm com os dados.

\subsubsection{Inferência Bayesiana}

Para Hainline (2013), a inferência estatística moderna pode ser dividida em duas principais escolas de pensamento: frequentista e bayesiana.

A abordagem frequentista é o método clássico de análise estatística em que informação prévia, obtida por estudos ou ensaios anteriores, é usada apenas durante a fase de planejamento. Estatística Bayesiana, ao contrário, constrói informação prévia para a análise formal, uma vez que se torna disponível. Ensaios anteriores, estudos em outros países, ou opiniões de especialistas são considerados fontes válidas de informação prévia. 0 uso de informação prévia na análise pode ser útil no planejamento do estudo e pode-se argumentar que um resultado mais preciso resulta de uma análise bayesiana. A informação prévia permite ao pesquisador a diminuir o alcance de um ensaio, o que resulta em um resultado mais específico (HAINLINE, 2013).

Em uma análise frequentista, a interpretação dos dados depende das intenções do pesquisador. Para frequentistas, antes que o experimento seja conduzido, valores críticos devem ser determinados, e p-valores são a base para a tomada de decisões. Estatísticos bayesianos afirmam que as hipóteses devem ser comparadas com o quão bem elas explicam os dados. P-valores representam a probabilidade de observar uma estatística de teste igual ou mais extrema do que a observada, supondo que a hipótese nula é verdadeira. (HAINLINE, 2013).

Um p-valor pequeno em uma análise frequentista resultará na rejeição da hipótese nula (muitas vezes assumindo que não há associação) em favor da hipótese alternativa (muitas vezes alegando associação está presente). Estatística 
bayesiana permite ao pesquisador obter odds ratio e probabilidades de previsão que possam demonstrar a magnitude do efeito que a variável tem sobre a variável de desfecho (HAINLINE, 2013).

Com análise bayesiana, cada análise compõe-se de dois tipos de informações: os dados que estão sendo analisados e a informação prévia. Sendo assim, o pesquisador é livre para escolher qualquer informação prévia que ajuda a explicar os dados que estão sendo analisados (HAINLINE, 2013).

Para Diggle e Ribeiro (2007), a inferência bayesiana é o processo de se encontrar um modelo de probabilidade para um conjunto de dados e resumir o resultado para uma distribuição de probabilidade sobre os parâmetros do modelo, que são tratadas como variáveis aleatórias e sobre quantidades não observadas como valores de novas observações (preditivas).

O teorema de Bayes é usado na inferência estatística para atualizar estimativas $d a$ probabilidade de que diferentes hipóteses sejam verdadeiras, baseado nas observações e no conhecimento de como essas observações se relacionam com as hipóteses (SORENSEN, 1996).

Para Amaral e Inácio (2010), os objetivos da estatística bayesiana são:

- representar o desconhecimento a priori sobre os parâmetros do modelo com uma distribuição de probabilidade (distribuição a priori);

- atualizar esse desconhecimento a priori com dados atuais - verossimilhança (likelihood);

- e produzir uma distribuição de probabilidade para o parâmetro que contenha menos desconhecimento (distribuição posteriori).

Segundo Bernardo (2001), a estatística bayesiana utiliza a probabilidade como uma medida condicional de incerteza associada com a ocorrência de um determinado evento, dada a informação disponível e os pressupostos aceitos.

Para Goşoniu (2008), a abordagem Bayesiana para inferência:

"permite estimativa de parâmetros utilizando informações provenientes dos dados através da função de probabilidade, bem como informações provenientes de outras fontes antes vistos os dados (ou seja, estudos anteriores, julgamentos subjetivos), que é formalizada através de distribuições anteriores. Teorema de Bayes combina a função de verossimilhança e a distribuição prévia para definição de uma nova quantidade, conhecida como distribuição posterior que forma a base da inferência Bayesiana. Os parâmetros são considerados como aleatórios $e$ as suas estimativas resultam não só num único valor, mas nas probabilidades de seus valores possíveis que são dadas pela sua distribuição de probabilidade, conhecida como a distribuição marginal posterior. "

Para Resende (2000), em inferência Bayesiana, certos métodos que assumem distribuições a priori não informativas, são essencialmente de inferência verossimilhança, tais como o método VEIL (ou da verossimilhança integrada de Gianola e Foulley, 1990) de estimação de componentes de variância, os quais mantêm a propriedade de conduzir a análise exata de amostra de tamanho finito.

Para Lavine (2000), a análise Bayesiana utiliza a distribuição posterior para resumir o estado de conhecimento. A distribuição posterior combina informações a partir dos dados na mão expressa através da função de verossimilhança, com outras informações expressas através da distribuição prévia.

\subsection{TÉCNICAS DE KRIGAGEM}

A origem da palavra krigagem vem do nome Daniel G. Krige, que foi o primeiro a introduzir - uso de médias móveis para evitar a superestimação sistemática de reservas de mineração (DELFINER e DELHOMME, 1975).

Segundo Câmara (2004), num primeiro momento o método da krigagem foi desenvolvido para resolver os problemas de mapeamentos geológicos, mas seu uso foi se expandindo com sucesso no mapeamento de solos, mapeamento hidrológico, mapeamento atmosférico e outros campos correlatos.

O método de Krigagem tem por fundamento a Teoria da Variável Regionalizada 
(TVR), desenvolvida por Matheron (1965). Uma variável regionalizada é uma variável distribuída no espaço ou tempo cujos valores são considerados como realizados de uma função aleatória.

Dantas (2003) diz que esta teoria identifica que a distribuição espacial de uma variável é expressa pela soma de três componentes:

- uma componente estrutural, tendo uma média constante ou tendência;

- uma componente aleatória espacialmente correlacionada, também chamada de variação regionalizada;

- uma componente aleatória não correlacionada espacialmente (erro residual).

O valor de uma variável $Z$, em uma posição geográfica $x$, representado por $Z(x)$, fica definido conforme a Equação 9 :

$$
Z(x)=m(x)+\varepsilon^{\prime}(x)+\varepsilon^{\prime \prime}
$$

\section{Em que:}

$\mathrm{m}(\mathrm{x})$ - é uma função determinística descrita pela componente estrutural de $Z$ em $x$;

$\varepsilon^{\prime}(\mathrm{x})$ - é a variação regionalizada;

$\varepsilon^{\prime \prime}$ - o resíduo do modelo, do tipo gaussiano, espacialmente independente, com média zero e variância constante $\sigma^{2}$.

Para Câmara (2003), a Krigagem:

"engloba um conjunto de métodos de estimação, incluindo procedimentos estacionários (krigagem simples $e$ ordinária), não estacionários (krigagem universal, funções intrínsicas de ordem k),

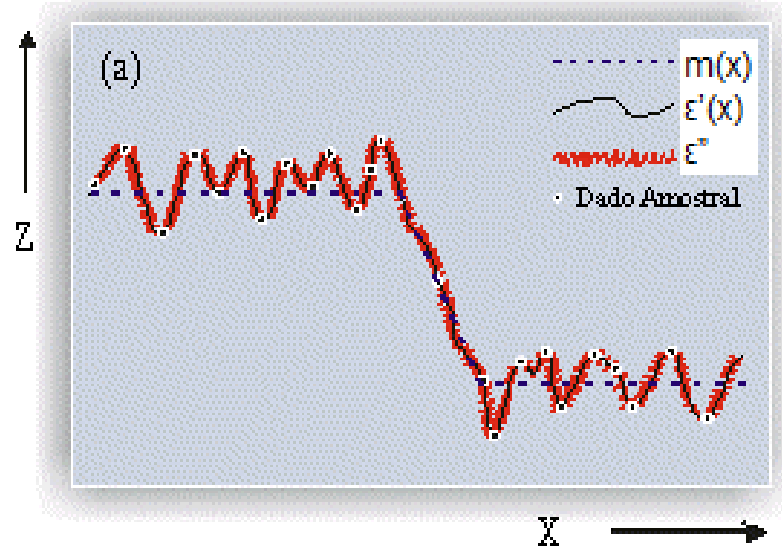

[a] univariados e multivariados (co-krigagem etc.)"

Para Soares (2002) a krigagem é:

"É o uso de médias móveis para evitar a superestimação. Ela difere de outros métodos de interpolação pela maneira como os pesos são atribuídos às diferentes amostras. $\mathrm{Na}$ krigagem, o procedimento é semelhante ao de interpolação por média móvel ponderada, exceto que os pesos são determinados a partir de uma análise espacial, baseada no semivariograma experimental."

A krigagem linear engloba um conjunto de métodos de estimação.

Segundo Soares (2002) a Krigagem simples foi inicialmente utilizada como um estimador de valores de atributos numéricos, em posições não observadas, para mapeamentos por médias ponderadas dos valores existentes das amostras locais conforme a Equação 10.

$$
\hat{Z}_{x 0}=m+\sum_{i=1}^{n} \lambda_{i}\left[Z\left(x_{i}\right)-m\right] \quad \text { Eq. [10] }
$$

Sendo o valor desconhecido que pode ser estimado a partir de uma combinação dos $n$ valores observados, $\mathrm{m}$ é a média que supõe-se que é conhecida a priori e $\lambda i$ são os pesos obtidos a partir do seguinte sistema de equações, denominado sistema de krigagem simples (CARVALHO, 1997):

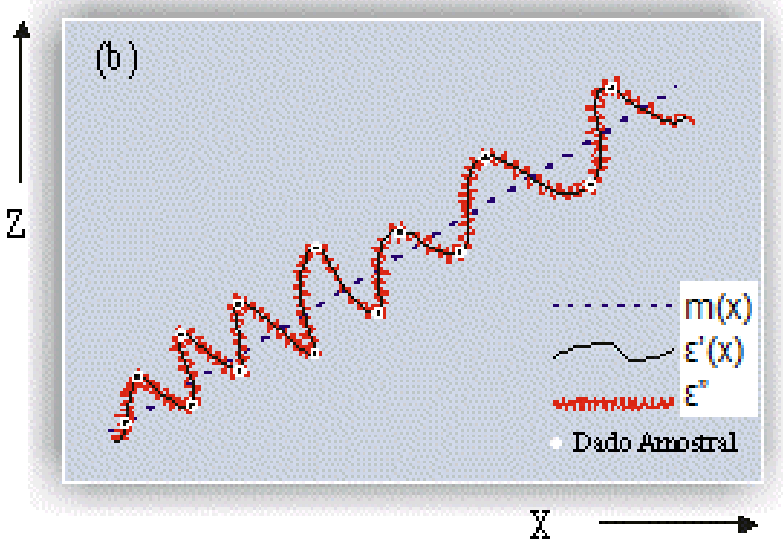

[b]

FIGURA 2: Distribuição espacial de uma variável, com componente estrutural [a] com média constante e [b] com tendência.

FONTE: Burrough (1987). 


$$
\begin{aligned}
& \underset{j=1}{n} \lambda_{i} C\left(x_{i}, x_{j}\right)=C\left(x_{i}, x_{0}\right) \text { para } \mathrm{i}=1, \ldots, \mathrm{n}(\mathrm{n} \\
& \text { equações) }
\end{aligned}
$$

\section{Em que:}

$C\left(x_{i}, x_{j}\right)$ refere-se à função covariância correspondente a um vetor, $h$, com origem em $x_{i} e$ extremidade em $\mathrm{x}_{\mathrm{j}}$;

$C\left(x_{i}, x_{0}\right)$ refere-se a função covariância correspondente a um vetor, $h$, com origem em $x_{i} e$ extremidade no ponto a ser estimado $\mathrm{x}_{0}$.

\section{APLICAÇÃO DO MÉTODO PROPOSTO}

A aplicação do método proposto foi realizada no bairro Centro do município de Balneário Camboriú localizado no Vale do Itajaí do Estado de Santa Catarina.

\subsection{PESQUISA DE MERCADO}

A pesquisa constituiu em um levantamento junto a imobiliárias da região de estudo, entrevistas com corretores, anúncios em classificados de jornais locais e pesquisa de campo in loco.

\subsubsection{Base de Dados}

Os dados foram coletados em Balneário Camboriú/SC, sendo que está coleta foi realizada no segundo semestre de 2009 considerando os imóveis transacionados (à vista, parcelado ou financiado) ou oferta. Inicialmente a base continha 120 dados, com transações e ofertas, diferentes padrões construtivos e diversas variáveis que serão descritas a seguir.

\subsubsection{Definição das variáveis e pressupostos}

Foram utilizadas diversas variáveis que são extraídas diretamente do banco de dados coletado, sendo algumas descartadas ao longo das modelagens por não se encaixarem no modelo ou devido à falta de importância na área de estudo.

Endereço: localidade física do dado, contendo a via e número no qual o mesmo se encontra, se disponível, pode contar o número do apartamento e a vaga vinculada a mesma, não fazendo parte do modelo matemático-estatístico;

Edifício: nome do residencial no qual se encontra o dado, não fazendo parte do modelo matemático-estatístico;

E e N: coordenadas métricas (Universal Transversa de Mercator - UTM) do dado representando a localização geográfica do imóvel, sendo que o datum usado foi WGS84;

Valor Total: variável quantitativa que representa o preço total do bem à venda em $\mathrm{R}$. Entretanto esta variável não foi utilizada uma vez que a variável Valor Unitário permite maior riqueza das informações e conclusões;

Área Total: variável quantitativa, em metros quadrados, da unidade, incluindo a área comum, que busca identificar a variabilidade dos preços em função da área total do imóvel. Caso tenha vagas de garagem em matrículas separadas, as áreas destas são incorporadas à área total da unidade;

Área Privativa: variável quantitativa, em metros quadrados, da unidade, não incluindo as áreas comuns e as áreas de vagas de garagem;

Número Total de Dormitórios: variável quantitativa que representa o número total de dormitórios, inclusive as suítes. Não estão sendo considerados os dormitórios de empregada. A premissa é que aumentando o número de quartos, aumenta o valor unitário do imóvel, podendo também ocorrer o contrário;

Número de Quartos de Empregada: variável quantitativa que representa o número total de quartos de empregada. Neste caso, não são considerados os demais dormitórios. A premissa é que com o aumento do número total de quartos de empregada implique em um valor unitário maior, podendo também ocorrer o contrário;

Número de Banheiros: variável quantitativa que representa o número total de banheiros. A premissa é que com o aumento do número total de banheiros implique em um valor unitário maior, podendo também ocorrer o contrário;

Quantidade de Vagas de Garagem: variável quantitativa que representa o número total vagas de garagem coberta e descobertas associadas ao apartamento, com ou sem matrícula independente. A premissa é que com o aumento do número total de vagas de garagem implique em um 
valor unitário maior;

Quantidade de Elevadores: variável quantitativa que representa o número total de elevadores. A premissa é que com o aumento do número total de elevadores implique em um valor unitário maior;

Fachada com Revestimento: variável dicotômica que busca identificar a variabilidade dos preços dos imóveis em razão da fachada com revestimento no edifício, sendo que a inexistência recebe o valor 0 (zero) e 1 (um) pela existência. A premissa é que a existência de fachada com revestimento nas edificações agregue valor ao imóvel.

Idade: variável quantitativa, em anos, que representa a idade real e/ou aparente da edificação. A premissa é que imóveis mais antigos, ou não reformados, tenham seus valores de venda menores;

Andar: variável quantitativa que representa o pavimento em que se encontra a unidade, buscando identificar a variabilidade dos preços conforme a altura da unidade na edificação. E a premissa é que unidades em pavimentos mais elevados possuam o valor de venda maior;

Vista Para o Mar: variável dicotômica que busca identificar a variabilidade dos preços dos imóveis em razão da existência de vista para o mar no apartamento, sendo que a inexistência recebe o valor 0 (zero) e 1 (um) pela existência. A premissa é que a existência de vista para o mar agregue valor ao imóvel.

Sacada com Churrasqueira: variável dicotômica que busca identificar a variabilidade dos preços dos imóveis em razão da existência de sacada com churrasqueira no apartamento, sendo que a inexistência recebe o valor 0 (zero) e 1 (um) pela existência. A premissa é que a existência de sacada com churrasqueira agregue valor ao imóvel;

Frente ou Fundos: variável dicotômica que busca identificar a variabilidade dos preços dos imóveis em razão do apartamento ser de frente ou de fundos, sendo que se for de fundos recebe o valor 0 (zero) e 1 (um) se for de frente. Tem-se como premissa que o apartamento de frente seja mais valorizado;

Piscina: variável dicotômica que busca identificar a variabilidade dos preços dos imóveis em razão do edifício ter piscina no condomínio, sendo que se não tiver recebe o valor 0 (zero) e 1 (um) se tiver. A premissa que 0 apartamento localizado em um condomínio que tenha piscina seja mais valorizado;

Evento: variável dicotômica que procura representar a natureza do evento, sendo valor 1 (um) para transação e valor 0 (zero) para oferta de mercado. Adotou-se a premissa de que possa haver uma diferença, para menor, entre o valor ofertado e o efetivo.

Valor Unitário: variável quantitativa que expressa o valor unitário, em $\mathrm{R} \$ \mathrm{~m}^{2}$, dos imóveis pesquisados. E a premissa é que o aumento da área do imóvel diminua o valor unitário;

COD: informação meramente ilustrativa que representa $o$ número escolhido para representar o edifício em questão para facilitar a sua localização no mapa da região, não fazendo parte do modelo matemático-estatístico;

Distância ao Pólo: variável quantitativa, em metros, que busca identificar a variabilidade dos preços dos dados pesquisados em função de sua distância até o Pólo de referência. A premissa é que o aumento da distância do imóvel em relação ao Pólo de referência provoque uma diminuição do valor unitário. No caso, os pólos que foram testados são: Distância da Praia e Distância da Praça Tamandaré;

Padrão: a variável padrão de acabamento descreve os vários níveis de acabamento de um edifício. Utilizou-se da variável qualitativa, pontuando (1) baixo; (2) normal e (3) alto. Para este caso adotou-se a premissa de que os valores aumentam conforme melhora $o$ padrão de acabamento;

Estado de Conservação: define como uma variável qualitativa utilizada para avaliar o estado geral do imóvel, pontuando Ruim (1), Médio (2), Bom (3), Ótimo (4). Para este caso adotou-se a premissa de que os valores aumentam conforme o seu melhor estado de conservação.

\subsubsection{Saneamento dos dados}

Inicialmente o banco de dados continha 120 dados, contendo ofertas e transações de 
mercado, foi realizado um saneamento retirando dados com valores incoerentes, além dos que não condiziam com a realidade do local, entre outras inconsistências, restando 104 dados.

\subsubsection{Variáveis usadas no modelo de regressores}

As variáveis independentes que mostraram serem significativas para a avaliação na área onde foi aplicado o método estudado são as seguintes: a) Área Total (AT) medida em $\mathrm{m}^{2}$; b) Frente ou Fundos (Frente), sendo 0 apartamento de fundos e 1 de frente; c) Andar; d) Idade; e) Número de Dormitórios (ND); e f) Distância da Praia (DPRAIA) - medido pelo caminho até a praia e não em linha reta.

Como variável dependente para os modelos foi definida o Valor Unitário (VU), medido em reais por metro quadrado.

\subsection{RESULTADO E ANÁLISES}

\subsection{1 modelos de regressão}

Para encontrar um modelo de regressão que melhor explique o valor da localização na área em estudo, buscou-se uma equação com as variáveis significativas na formação do valor para os imóveis.

Foram feitas transformações das variáveis independentes e da variável dependente, e também foram analisadas as interações entre as variáveis independentes para encontrar o melhor modelo de regressão.

\subsubsection{Regressão linear múltipla para as variáveis formadoras de valor dos apartamentos}

Foram testadas várias equações de regressão para as variáveis, visando encontrar um modelo que possa explicar melhor a formação do valor dos apartamentos no bairro Centro do município de Balneário Camboriú/SC.

O melhor modelo de regressão encontrado pode ser observado na Equação 10:

$$
\begin{gathered}
\ln (V U)=5,511401+(31,12281 \times(1 / \mathrm{AT})) \\
+(2,70535 \times(1 / \text { DPRAIA }))+\left(0,6073081 \times \mathrm{ND}^{1 / 2}\right) \\
+(-0,01493818 \times \text { Idade })+(0,01416053 \times \text { Andar }) \\
+(0,1918692 \times \text { Frente })
\end{gathered}
$$

Em que:

$\mathrm{VU}=$ valor unitário $\left(\mathrm{R} \$ \mathrm{~m}^{2}\right)$;

$A T=$ área total $\left(\mathrm{m}^{2}\right)$;

DPRAIA = distância da praia pela estrada $(m)$;

$\mathrm{ND}^{1 / 2}=$ número de dormitórios;

Andar = andar do apartamento;

Idade = idade do apartamento;

Frente $=$ apartamento de frente ou de fundos .

O Quadro 1 apresenta os valores e estatísticas referentes ao coeficiente de determinação, à significância dos regressores, ao sinal, e também à significância da regressão.

Pode-se observar que os coeficientes de todas as variáveis são significativos, com o nível de significância menor que 5\%, com coeficiente de determinação superior a $78 \%$, e o modelo sendo classificado como grau III neste requisito segundo a NBR 14.653-2 (ABNT, 2011).

Os sinais dos regressores são coerentes com o comportamento esperado no mercado imobiliário local.

\subsubsection{Regressão espacial}

\subsubsection{Estimação da matriz de pesos}

Na regressão espacial foi utilizada a matriz de vizinhança de até 300 metros, pois foi a que melhor explicou a formação do valor dos imóveis do município de Balneário Camboriú/SC no Bairro Centro, como pode ser observado no semivariograma da Figura 3:

Como pode ser observado no semivariograma experimental omnidirecional da Figura 3 os dados apresentam maior dependência espacial até uma distância aproximadamente de 300 metros, e depois desta distância a dependência diminui consideravelmente apresentando uma estrutura praticamente estacionária. Desta maneira, pode se notar que os imóveis que se encontram muito próximas exercem uma forte influência entre si e esta influência diminui consideravelmente com a distância, de forma que, aqueles imóveis que se encontram separados por distâncias maiores quase não apresentam influência entre si. 
QUADRO 1: Valores da regressão para o modelo das variáveis de formação dos valores.

\begin{tabular}{|c|c|c|c|}
\hline & Coeficiente & Significância \\
\hline Variáveis independentes & Constante & 5,511401 & 0,000000000 \\
\hline & 1/AT & 31,12281 & 0,014505200 \\
\hline & 1/DPRAIA & 2,70535 & 0,000000000 \\
\hline & $\operatorname{ND}^{\wedge}(1 / 2)$ & 0,6073081 & 0,000012700 \\
\hline & Andar & 0,01416053 & 0,000156500 \\
\hline & Idade & -0.01493818 & 0,000000300 \\
\hline & Frente & 0,1918692 & 0,001342800 \\
\hline Variável dependente & $\ln (\mathrm{VU})$ & & \\
\hline $\mathbf{R}^{\mathbf{2}}$ & 0,784242 & & \\
\hline$R^{2}$ ajustado & 0,770896 & & \\
\hline F-estatístico & 58,763 & & \\
\hline Prob (F-estatístico) & $3,87 \mathrm{E}-25$ & & \\
\hline Erro padrão estimado & 0,169293 & & \\
\hline Números de observações & 104 & & \\
\hline
\end{tabular}

FONTE: Autoria própria.

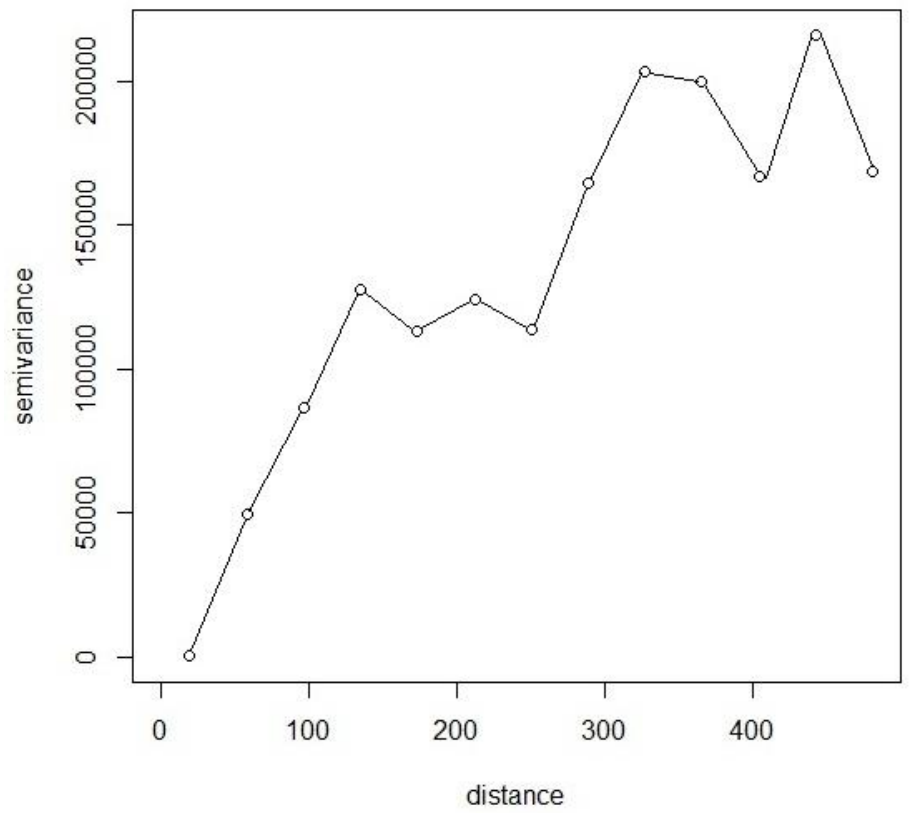

FIGURA 3: Semivariograma experimental omnidirecional ou isotrópico do VU. FONTE: Autoria própria.

\subsubsection{Testes de autocorrelação espacial}

Foram calculadas as estatísticas correspondentes aos testes de autocorrelação espacial de Moran's I, e também os testes do Multiplicador de Lagrange ( $L M$ ) com a matriz $W$ (do peso), definida para o modelo da variável dependente e do erro e suas estatísticas robustas. No Quadro 2 podem-se observar os resultados.
QUADRO 2: Testes de autocorrelação espacial do modelo.

\begin{tabular}{|l|c|c|}
\hline TESTE & VALOR & PROBABILIDADE \\
\hline Moran's I & 0,8372797 & 0,4024352 \\
\hline LM (lag) & 6,0234965 & 0,0141167 \\
\hline LM robusto (lag) & 5,8629350 & 0,0154630 \\
\hline LM (erro) & 0,2933405 & 0,5880878 \\
\hline LM robusto (erro) & 0,1327790 & 0,7155679 \\
\hline
\end{tabular}


Esses resultados mostram que há uma autocorrelação espacial nos resíduos do modelo de regressão por mínimos quadrados, pois a probabilidade do teste $L M$ (lag) é menor que $5 \%$. Sendo assim, o modelo da defasagem espacial apresenta-se significativo.

No modelo de defasagem espacial foi usado a matriz de até 300 metros, pois foi a que melhor explicou a formação do valor dos apartamentos do município de Balneário Camboriú/SC no bairro centro.

No Quadro 3 podem ser observados os resultados encontrados.

O coeficiente autorregressivo espacial é estimado como 0,02911609, e é significativo $(p=0,0110207)$.

Segundo Trivelloni (2005), a estatística $z$ corresponde ao equivalente para a regressão por máxima verossimilhança ao valor $t$ de Student para o método de mínimos quadrados. As probabilidades indicam o grau de significância de cada variável de forma análoga que na regressão por mínimos quadrados

\subsection{KRIGAGEM PARA AVALIAÇÃO EM MASSA DE IMÓVEIS}

Para estimar o valor unitário dos apartamentos, foi feito uma homogeneização, sendo que para isso foram usados três apartamentos paradigmas conforme 0 Quadro 4.

Após se ter encontrado o modelo de predição que melhor correspondeu com a realidade para a praia central de Balneário Camboriú/SC, foi feita uma krigagem ordinária e uma krigagem bayesiana.

Usando os Valores Unitários calculados com o modelo de regressão na defasagem foi construído um semivariograma experimental conforme Figura 4.

Pode-se observar que o gradiente de variação se estabiliza aproximadamente a partir dos 300 metros de distância, e depois desta distância a dependência diminui consideravelmente apresentando uma estrutura praticamente estacionária.

Em seguida para os mesmo Valores Unitários foi feita uma Krigagem Bayesiana para os imóveis avaliandos conforme Figura 5. Podem ser observadas as áreas com maior e menor valor conforme o gradiente de cores que permite uma análise visual mais rápida e simples das áreas.

Na Figura 6 a área avaliada foi separada em três setores $A, B$ e $C$, sendo assim pode-se identificar ainda melhor a variação de valores.

\begin{tabular}{|c|c|c|c|c|}
\hline Variável & Coeficiente & Erro Padrão & Valor Z & Probabilidade \\
\hline W_In(VU) & 0,0291161 & 0,0114538 & 2,5420430 & 0,0110207 \\
\hline Constante & 5,3072010 & 0,2747589 & 19,3158500 & 0,0000000 \\
\hline $1 /(\mathrm{AT})$ & 34,9974700 & 11,8274100 & 2,9590130 & 0,0030864 \\
\hline 1/(DPRAIA) & 2,7882940 & 0,2047816 & 13,6159400 & 0,0000000 \\
\hline ND & 0,5909657 & 0,1238395 & 4,7720290 & 0,0000018 \\
\hline Andar & 0,0140154 & 0,0033739 & 4,1541190 & 0,0000327 \\
\hline Idade & $-0,0158776$ & 0,0025567 & $-6,2101900$ & 0,0000000 \\
\hline Frente & 0,1930556 & 0,0544589 & 3,5449760 & 0,0003927 \\
\hline \multicolumn{5}{|c|}{$\begin{array}{l}\text { Legenda: } \\
\text { VU = valor unitário }\left(\mathrm{R} \$ / \mathrm{m}^{2}\right) ; \\
\text { AT = area total }\left(\mathrm{m}^{2}\right) ; \\
\text { DPRAIA = distância da praia ao longo da rua }(\mathrm{m}) ; \\
\text { ND = número de domrintórios; } \\
\text { Andar = andar do apartamento; } \\
\text { Idade = idade do apartamento; } \\
\text { Frente = apartamento de frente ou de fundos. }\end{array}$} \\
\hline
\end{tabular}




\begin{tabular}{|c|c|c|c|c|c|}
\hline \multicolumn{7}{|c|}{ QUADRO 4: Apartamentos paradigmas. } \\
\hline Paradigma & Frente & Andar & Idade & ND & AT \\
\hline 1 & 1 & 4 & 6 & 3 & 238,65 \\
\hline 2 & 0 & 5 & 5 & 3 & 170,00 \\
\hline 3 & 1 & 3 & 12 & 4 & 156,00 \\
\hline
\end{tabular}

FONTE: Autoria própria.

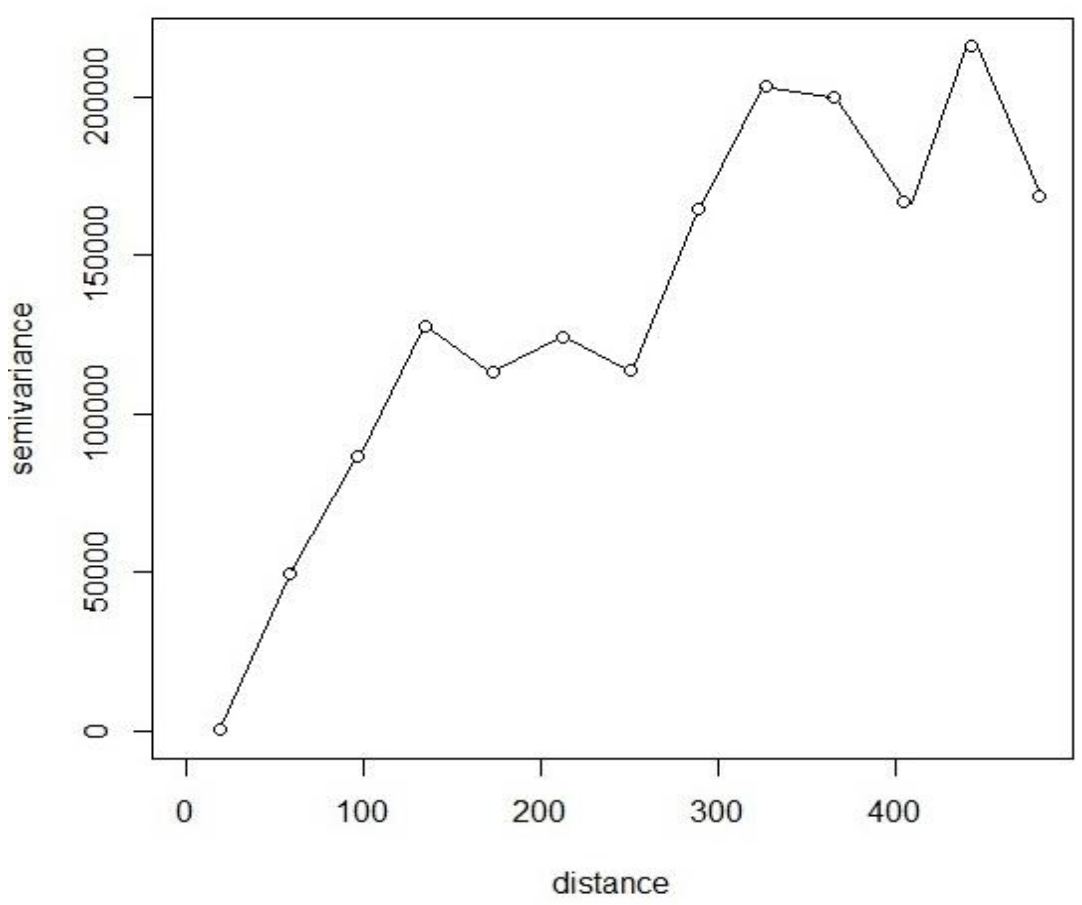

FIGURA 4: Semivariograma experimental para o VU.

FONTE: Autoria própria.

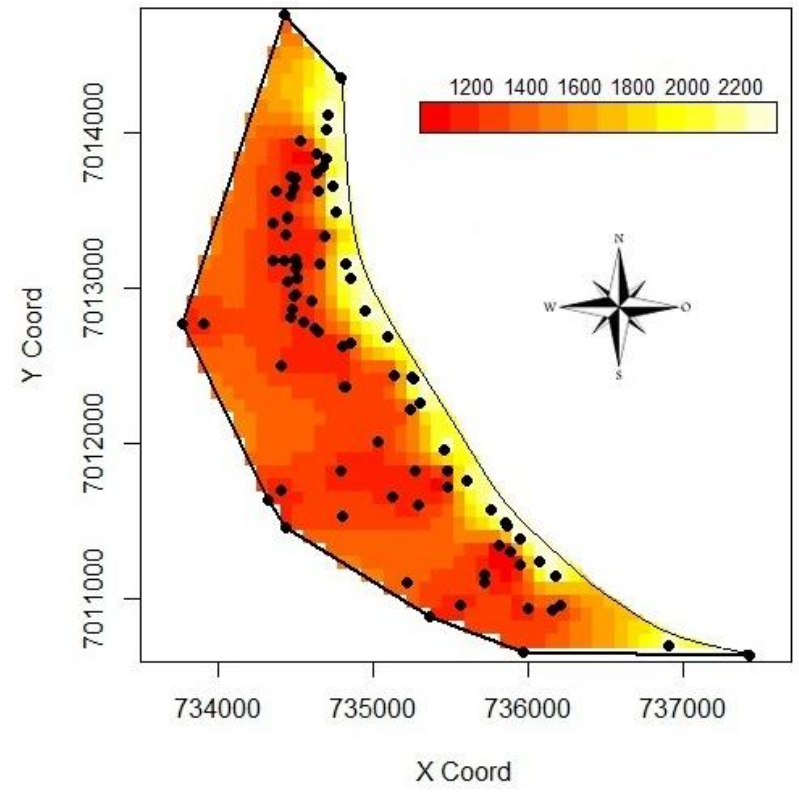

FIGURA 5: Krigagem para variável VU usando método SLM para os imóveis avaliandos. FONTE: Autoria própria.

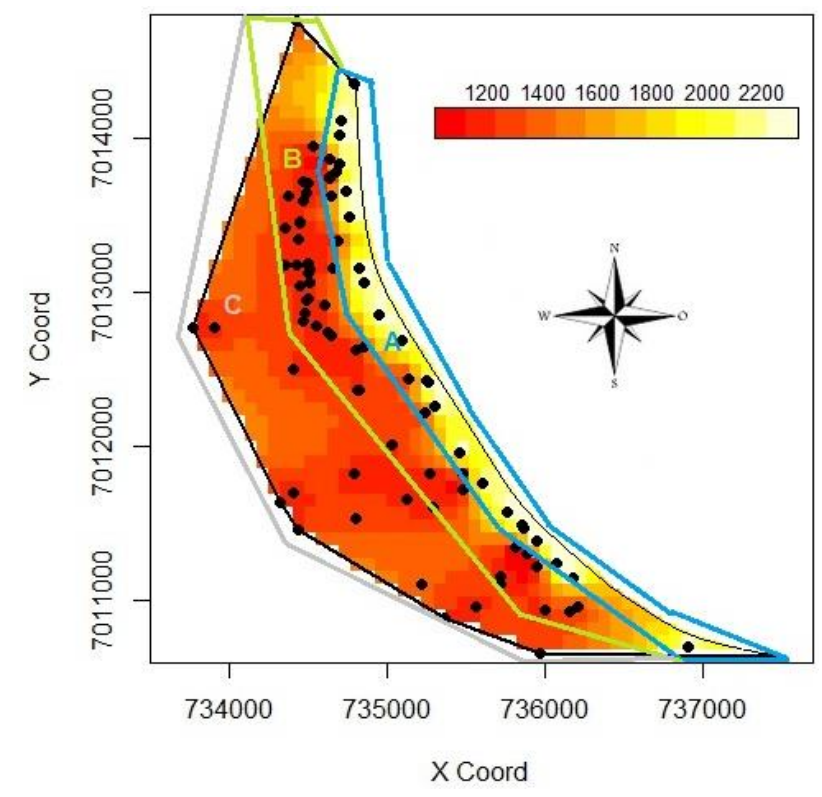

FIGURA 6: Krigagem para variável VU usando método SLM para os imóveis avaliando dividida em três setores. FONTE: Autoria própria. 
A área em tom amarelado, que pode ser observada no setor $A$, são as áreas mais valorizadas, pois são aquelas de frente ao mar e proximidades.

Entretanto as áreas em tom avermelhadas, os setores $B$ e $C$, são aquelas áreas que ficam mais de 300 metros da praia, sendo que quanto mais afastado for o imóvel (setor C), o valor do metro quadrado diminui. $O$ setor $C$ é aquele que fica próximo a $B R$ 101. Porém não diminui de forma acentuada, pois os imóveis localizados na área em que o modelo foi aplicado existem pouca variação de valor.

$\mathrm{Na}$ Figura 7, pode-se observar que valores do setor B entram também no setor $C$. Isto pode ser observado em três pontos diferentes do setor C. Nestes lugares há uma grande concentração comercial.

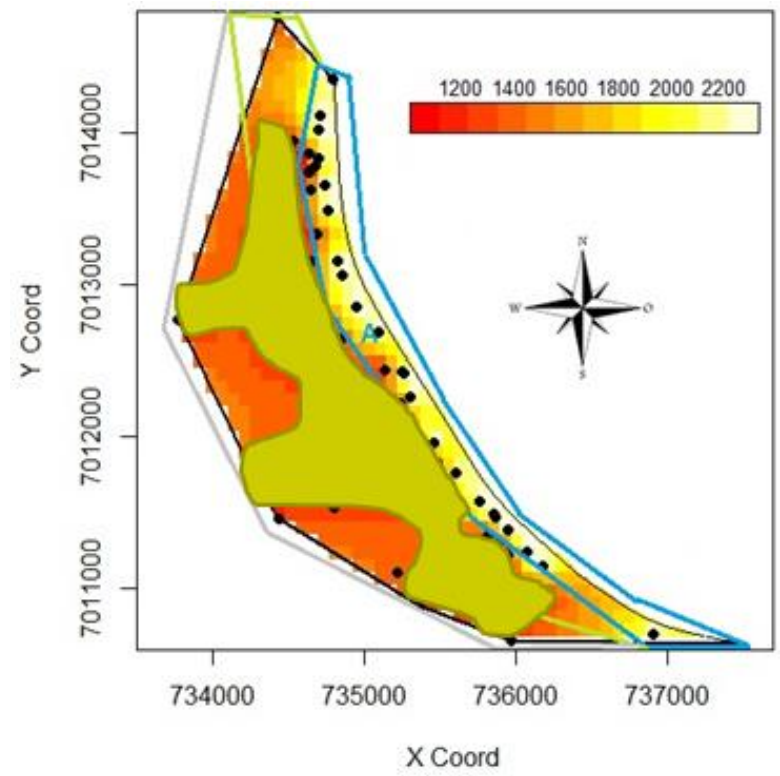

FIGURA 7: Krigagem para variável VU usando método SLM para os imóveis avaliando dividida em três setores.

FONTE: Autoria própria.

A cidade de Balneário Camboriú como um todo, é uma cidade valorizada e com crescimento nos valores dos imóveis nos últimos 10 anos.

Por fim, os resultados da Krigagem da Figura 7 apresentaram coerência com a realidade da área de estudo possibilitando uma fácil interpretação.

\section{CONCLUSÕES}

O método de regressão $S L M$ utilizado comprovou ser adequado pelos testes de autocorrelação espacial nos resíduos do modelo de mínimos quadrados calculados com a matriz $W$ serem significativos e pela significância dos modelos de regressão espacial estimados.

O semivariograma experimental mostrou ser uma ferramenta muito importante para a construção da matriz de pesos espaciais para a regressão espacial, definindo assim o principal parâmetro da matriz que é a distância máxima de vizinhança em função da análise dos dados de mercado.

A krigagem bayesiana obteve resultados condizentes com a realidade do local, sendo assim, serviu para gerar valores entre os vizinhos, pois dificilmente se obtém dados na coleta em campo para toda a área estudada. Entretanto deve ser lembrado que a krigagem é um método de interpolação, que vizinhos muito afastados podem gerar erros na estimação dos valores unitários.

Uma importante contribuição deste trabalho foi o uso combinado da geoestatística e da krigagem bayesiana, pois não eram conhecidos estudos similares antes do desenvolvimento desta pesquisa.

Os resultados foram significantes, pois a estatística espacial ajudou a encontrar o modelo de predição de valores unitários condizentes com a realidade do local.

Uma importante contribuição deste trabalho foi o uso combinado da econometria espacial e da geoestatística bayesiana, usando as técnicas da regressão espacial e da krigagem bayesiana. Estudos similares ainda não tinham sido desenvolvidos para a avaliação em massa de imóveis. A combinação destas técnicas possibilitou a elaboração de uma planta de valores que refletiu bem a realidade do mercado imobiliário do local de estudo. Assim, este trabalho apresenta uma importante contribuição para a determinação do valor justo dos imóveis numa avaliação em massa. Desta maneira, o método proposto mostra-se como importante ferramenta para a determinação de valores que permitirão uma melhor equidade $e$ consequente justiça fiscal quando aplicados pelos municípios. 


\section{REFERÊNCIAS BIBLIOGRÁFICAS}

ABNT (ASSOCIAÇÃO BRASILEIRA DE MORMAS TECNICAS) - Avaliação de bens - parte 2: imóveis urbanos: Norma NBR - 14.653-2/2011, Rio de Janeiro: ABNT, 2011.

ANDRADE, P. J. N. Sistemas Especialistas de Apoio ao Diagnóstico em Medicina. Relações com o Teorema de Bayes e com a Lógica do Raciocínio Diagnóstico. Fortaleza, 1999. Disponível em: < http://publicacoes.cardiol.br/abc/1999/7306/73060008. pdf>. Acessado em 21 outubro de 2014.

AMARAL, E. F. L. e INÁCIO, M. M. Modelos Bayesianos. Belo Horizonte, 2010. Disponível em: <http://www.ernestoamaral.com/docs/dcp859b4-

102/Aula142.pdf>. Acessado em: 15 março de 2014.

ANSELIN, L. Spatial Econometrics. Discussion paper. Bruton Center, School of Social Sciences, University of Texas at Dallas, 1999a.

AYRES, A. Como avaliar imóveis. São Paulo: Editora Imobiliária, 1996.

BERNARDO, J. M. Bayesian Statistics. Encyclopedia of Life Support Systems (EOLSS). Paris: UNESCO (to appear) 2001.

BRASIL, G. H. Minicurso Noções de Estatística Bayesiana. Universidade Federal do Espírito Santo (UFES) DEST/PPGECO/PPGGP, Vitória, 2012.

BURROUGH, P. A. Principles of geographical information systems for land resources assessment. Oxford, Clarendon Press, 1987.

CÂMARA, G., et al. Análise Espacial de Dados Geográficos. São José dos Campos, INPE, 2003 - on-line (3a. edição, revista e ampliada). Dezembro 2004. Disponível em: <http://www.dpi.inpe.br/gilberto/tutoriais/analise/> Acessado em: 10 de fevereiro de 2007.

CAN, A. GIS and Spatial Analysis of Housing and Mortgage Markets. Journal of Housing Research, v.9, s/n, 1998.

CARROLL, J. L. A Bayesian Decision Theoretical Approach to Supervised Learning, Selective Sampling, and Empirical Function Optimization. Tese de doutorado desenvolvida na Faculty of Brigham Young University, 2010. 357p.

CARVALHO, M. S. Aplicação de métodos de análise espacial na caracterização de áreas de risco a saúde. Tese Doutorado em Engenharia Biomédica. Rio de Janeiro, COPPE/UFRJ, 1997.

CESARE, C. de. Valuación de inmuebles para fines fiscales. Impuesto a la propriedade inmobiliaria. Lincoln Institute of Land Policy, 2004.
CRESSIE, N. A. C. Statistics for Spatial Data. Revised Edition, John Wiley \& Sons, Inc., 1993.

DANTAS, R. A. Modelos Espaciais Aplicados ao Mercado Habitacional um Estudo de Caso Para a Cidade do Recife. Tese de Doutorado, Universidade Federal de Pernambuco, Recife, 2003. 133p.

DELFINER, P.; DELHOMME, J. P. Optimum interpolation by Kriging. In: Davis, J. C.; McCullagh, M. J. ed. Display and analysis of spatial data. New York, John Wiley, 1975.

DIGGLE, P. J.; RIBEIRO JR., P. J. Model-Based geostatistics. New York: Springer, 2007. 228p.

DiMAGGIO, C. Spatial epidemiology notes applications and vignettes in R. Center for Injury Epidemiology and Prevention Columbia University, NY, 2014. 178p.

EHLERS, R. S. Introdução a Inferência Bayesiana. Universidade de São Paulo, São Paulo, Livro Digital, Editora USP. 2003.

GANGSEI, L. E. A Bayesian method for estimating moose (Alces alces) population size based on hunter observations and killed at age data. Dissertação de mestrado desenvolvida no Department of Chemistry, Biotechnology and Food Science, Norwegian University of Life Sciences, 2013.

GIANOLA, D.; FOULLEY, J. L. Variance estimation from integrated likelihood (VEIL). Genetics Selection Evolution, v.22, s/n, p.403-417, 1990.

GONZÁLEZ, M. A. S. Aplicação de Técnicas de Descobrimento de Conhecimento em Base de Dados e de Inteligência Artificial em Avaliações de Imóveis. Tese de Doutorado, Universidade Federal de Rio Grande do Sul, Programa de Pós-Graduação em Engenharia Civil, 2002. 296p.

GOŞONIU, L. Development of Bayesian geostatistical models with applications in malaria epidemiology. Tese de doutorado, Universität Basel, PhilosophischNaturwissenschaftlichen Fakultät, 2008. 166p.

HAINLINE, A. E. Frequentist and bayesian modeling in the presence of unmeasured confounding. Tese de doutorado, Faculty of Baylor University In Partial Fulfillment of the Requirements for the Honors Program. Waco, Texas May 2013. 56p.

LAVINE, M. What is Bayesian statistics and why everything else is wrong. Technical report, Duke University, North Carolina, 2000.

LEMOS, M. B. et al. A Organização Territorial da Indústria no Brasil. In: NEGRI, J. A.; SERGIO, M. (Orgs.). Inovações, Padrões Tecnológicos e Desempenhos das Firmas Industriais Brasileiras. Brasília: IPEA, 2005, p. 325-363.

LOPES, S. B.; BRONDINO, N. C. M.; SILVA, A. N. R. Análise Do Desempenho De Modelos De Regressão Espacial Na Previsão De Demanda Por Transportes. In: XIV Congreso 
Panamericano de Ingeniería de Tránsito y Transporte, 2006, Las Palmas de Gran Canaria. XIV PANAM, 2006.

MATHERON, G.: Les Variables Regionalisées et Leur Estimation, Masson, Paris. 1965.

MÖLLER, L. F. C. Planta de valores genéricos: avaliação coletiva de imóveis para fins tributários. Porto Alegre: Sagra-DC Luzzatto, 1995.

PAIVA, W. L., KHAN, A. S. DEPENDÊNCIA ESPACIAL E EMPREGO FORMAL: O que é possível afirmar para indústria cearense?. In. Anais do VI Encontro de Economia do Ceará em Debate. Fortaleza - CE, 05 de novembro de 2010.

PENA, S. D. Bayes: o 'cara'!. Ciência Hoje, Rio de Janeiro, v.38, n.228, p. 22 - 29, jul. 2006. Available in: <http://cienciahoje.uol.com.br/banco-de-

imagens/lg/protected/ch/228/bayes.pdf/at_download/f ile>. Access: 21 out. 2013.

POLI, C. R.; FRANCO, E. R.; ROCHA, R. J. de G.; AKIN, R. G. Planta de Valores Genéricos de Zona Residencial do Município de Fazenda Rio Grande - PR. XVI COBREAP Congresso Brasileiro de Engenharia de Avaliações e Perícias, Anais. Manaus, 2011.

RESENDE, M. D. V. de. Inferência Bayesiana e simulação estocástica (amostragem de Gibbs) na estimação de componentes de variância e de valores genéticos em plantas perenes. Colombo: Embrapa Florestas, 2000. 68p. (Embrapa Florestas. Documentos, 46).

SERRANO, R. M.; VALCARCE, E. V. Técnicas econométricas para el tratamiento de dados espaciales: la econométrica espacial. Edictions Universita de Barcelona, Barcelona, 2000.

SILVA, E. Cadastro técnico multifinalitário: base fundamental para avaliação em massa de imóveis. Florianópolis, 2006. Tese de doutorado em engenharia civil, Universidade Federal de Santa Catarina - UFSC, 220p.

SOARES, A. F. Uso do Módulo "Análise" do SPRING (Versão 3.2.02) Para Estudos de Dados Climáticos. Embrapa. Campinas - SP. 2002.

SORENSEN, D.; WANG, C. S.; JENSEN, J.; GIANOLA, D. Bayesian analysis of genetic change due to selection using Gibbs sampling. Genetic Selection Evolution, v. 26, s/n, p.333-360, 1994.

TRIVELlONI, C. A. P. Método Para Determinação do Valor da Localização Com Uso de Técnicas Inferências e Geoestatísticas na Avaliação Em Massa de Imóveis. Tese de Doutorado, Universidade Federal de Santa Catarina, Programa de Pós-Graduação em Engenharia Civil, 2005.

WETTIG, H. Probabilistic, Information-Theoretic Models for Etymological Alignment. Tese de doutorado, Faculty of Science of the University of Helsinki, 2013.
ZANCAN, E. C. Avaliações de Imóveis em Massa para Efeitos de Tributos Municipais. Edição única, Editora Rocha, Florianópolis, 1996. 121p. 